DOI: $10.26461 / 18.01$

\title{
Desarrollo y optimización de una metodología para la determinación de metales y semimetales en cabello
}

\author{
Development and optimization of a methodology \\ to determinate metals and semimetals in hair \\ RECIBIDO: 8/4/2019 $\rightarrow$ APROBADO: 20/5/2019 $\square$ fiaquinta@fq.edu.uy
}

i̊ iaquinta, fiorella (1); Álvarez, Cristina (2); COUsillas, adriana (2); MAÑAY, NELLY (2)

(1) Área de química analítica, Departamento estrella campos, FACULTAD DE QUímicA, UNIVERSIDAD DE LA REPÚBLICA, MONTEVIDEO, URUGUAY

(2) Cequimtox, ÁRea toxicología, departamento estrella CAMpos, FACUlTad DE QUímica, UNIVERSidAd DE LA REPÚblicA, MONTEVIDEO, URUGUAY

\section{RESUMEN}

La determinación de niveles de elementos traza en cabello como alternativa a otras matrices biológicas está siendo ampliamente utilizada en programas de biomonitoreo como biomarcadores de exposición crónica a contaminantes ambientales y para evaluar patologías asociadas al exceso o carencia de determinadas sustancias. El cabello es una matriz compleja para análisis de trazas que presenta importantes desafíos analíticos y preanalíticos. Se presenta el desarrollo y validación de una metodología para la determinación de mercurio $(\mathrm{Hg})$, plomo $(\mathrm{Pb})$, arsénico (As) y manganeso (Mn) por espectrometría de absorción atómica. La digestión de la muestra se realizó mediante digestión asistida por microondas. El mercurio se determinó utilizando vapor frío (CV-AAS) y la generación de hidruros (HGAAS) fue utilizada para el caso del arsénico. Las determinaciones de manganeso y plomo se realizaron por atomización electrotérmica (ET-AAS). Los parámetros de desempeño evaluados fueron rango lineal, límites de cuantificación y detección, precisión a tres niveles y veracidad. Este último fue evaluado utilizando un material de referencia certificado de cabello $\mathrm{n}^{\mathrm{o}} 13$ Human Hair, del Instituto Nacional de Estudios Ambientales (NIES) de Japón. La validación de esta metodología es de gran utilidad para el estudio de la exposición a estos analitos, dada la simplicidad de la obtención de la muestra.

PAlabras ClAVE: Pelo, metales, semimetales, toxicología, espectrometría. 


\section{ABSTRACT}

Hair is an alternative biological matrix widely used in biomonitoring programs. It is used as a biomarker of chronic exposure to environmental contaminants and to evaluate pathologies related to the excess or shortage of certain substances. Hair is a complex matrix for the evaluation of toxics in low levels. This matrix is an analytic and pre-analytic challenge. In this work, the development and validation of a methodology for the determination of mercury $(\mathrm{Hg})$, lead $(\mathrm{Pb})$, arsenic $(\mathrm{As})$ and manganese $(\mathrm{Mn})$ by atomic absorption spectrometry is presented. The sample treatment was carried out by microwave assisted digestion. The determination of $\mathrm{Hg}$ was carried out using cold vapor (CV-AAS) and hydride generation (HG-AAS) was used for As. Mn and $\mathrm{Pb}$ were analyzed by electrothermal atomization (ET-AAS). The figures of merit evaluated were linear range, limits of quantification and detection, accuracy at three levels and veracity. The latter was evaluated using a hair reference material (No. 13 Human Hair, from the National Institute of Environmental Studies-NIES, Japan). The validation of this methodology is very useful for the study of exposure to these analytes, because of the simplicity of sample obtention. KEYWORDS: Hair, metals, semimetals, toxicology, spectrometry.

\section{INTRODUCCIÓN}

A lo largo de la evolución, los organismos han incorporado elementos inorgánicos en sus sistemas biológicos. Dado que muchos de esos sistemas dependen de la presencia de estos elementos, se han considerado esenciales para el desarrollo de la vida. Un elemento es esencial cuando su ausencia o defecto causa problemas para el desarrollo del organismo, pero es capaz de revertirlos cuando se lo vuelve a incorporar en la dieta. La presencia de estos elementos debe ser equilibrada, ya que su exceso puede ser tóxico para la salud (Casarett, 2005).

El arsénico $(\mathrm{As})$, el plomo $(\mathrm{Pb})$ y el mercurio $(\mathrm{Hg})$ son los tres primeros elementos de la lista prioritaria de sustancias peligrosas debido a su frecuencia, toxicidad y potencial de exposición humana en sitios contaminados (ATSDR, 2017). Estos elementos se consideran tóxicos sistémicos que inducen múltiples lesiones de órganos y varias enfermedades, que van desde problemas neurológicos a pigmentaciones o irritaciones en la piel, incluso a niveles bajos de exposición.

En el caso del As, hay varios estudios que correlacionan la exposición inorgánica del As con el desarrollo de cáncer de pulmón y de piel (Mañay, 2013), lo que ha llevado a que la Agencia Internacional de Investigación contra el Cáncer (IARC) lo clasifique como cancerígeno para el hombre (IARC, 2018).

La toxicidad del plomo está ampliamente estudiada. Entre los efectos más característicos se encuentran la alteración de la síntesis del grupo hemo, que provoca la inhibición de la formación de hemoglobina, y problemas neurológicos. En el caso de los niños es una problemática de interés ya que provoca un comportamiento alterado, retraso mental, hiperactividad y sordera, entre otros trastornos. Estos efectos son 
debidos a que el plomo cruza la barrera hematoencefálica más fácilmente en niños que en adultos (Casarett, 2005).

La exposición al $\mathrm{Hg}$ puede causar insuficiencia renal y pulmonar, e incluso la muerte. En su forma metilada es un agente teratogénico, ya que es capaz de cruzar la barrera placentaria, causando malformaciones en el feto (Colangelo, 2013). Otros estudios que correlacionan la exposición al metal demuestran sus propiedades de mutagénesis. Algunos trabajos correlacionan la exposición al metal con aberraciones cromosómicas (Azevedo, et al., 2003).

Otros oligoelementos metálicos como el Mn son nutrientes esenciales requeridos para diversas funciones bioquímicas y fisiológicas en dosis adecuadas. Sin embargo, altas concentraciones de Mn pueden causar problemas neurológicos (Menezes-Filho, et al., 2009). La intoxicación por este metal puede afectar las funciones motoras, causando un síndrome similar al Parkinson (Tokar, 2013).

La concentración de estos elementos en cabello es útil como biomarcador de exposición en programas de biomonitoreo como screening, o mapeo de riesgos para la salud de la población (WHO, 2015). El cabello se puede utilizar como una muestra muy conveniente para la evaluación de la exposición crónica, lo que muestra su gran potencial como herramienta clínica de evaluación de exposición histórica a ciertas sustancias a lo largo del tiempo (ATSDR, 2001). Otro de los beneficios del uso de esta matriz es la sencillez de su obtención, ya que no es una muestra invasiva como la sangre. Varios estudios se han realizado con esta matriz, principalmente para evaluación a la exposición ambiental (Tirado, et al., 2015; Liang, et al., 2017), pero también en estudios entre niños con autismo y sus madres (Adams, 2006).

Desde el punto de vista analítico, al ser una matriz compleja presenta un gran desafío preanalítico, porque se debe asegurar que los resultados obtenidos corresponden exclusivamente a la excreción del organismo y no a la exposición exógena. Para ello es necesario establecer un riguroso protocolo de toma y lavado de la muestra.

A los efectos de contar con niveles de referencia de estos metales/metaloides, el máximo nivel de As en el pelo es $1 \mu \mathrm{g} \mathrm{g}^{-1}$ según la Agencia para las sustancias tóxicas y registro de enfermedades (ATSDR; ATSDR, 2007). Sin embargo, los niveles de Pb en cabello de referencia no están bien definidos porque cambian según la región de estudio. En Latinoamérica, algunos trabajos realizados en Venezuela y Chile consignaron niveles entre 5,28 y 7,70 $\mu \mathrm{g} \mathrm{g}^{-1}$ (Marcano, et al., 2009; Valdebenito, 2008). En el caso del $\mathrm{Hg}$, las Naciones Unidas sugieren niveles 1 a $2 \mu \mathrm{g} \mathrm{g}^{-1}$ para las personas que no han consumido pescado recientemente, mientras que valores de $10 \mu \mathrm{g} \mathrm{g}^{-1}$ pueden esperarse en personas que sí lo hicieron (Naciones Unidas, 2010).

En este trabajo se presenta el desarrollo, optimización y validación de una metodología para la determinación de As, $\mathrm{Pb}, \mathrm{Mn}$ y $\mathrm{Hg}$. La metodología requiere también la elaboración de un protocolo de muestreo y de preparación de muestras, que en este caso será asistida por microondas. Como se ha mencionado, esta matriz es ampliamente utilizada en programas de biomonitoreo, por lo cual su validación es de gran utilidad para evaluar los niveles de exposición poblacional. 


\section{MATERIALES Y MÉTODOS}

\section{Reactivos}

Las disoluciones se prepararon a partir de una disolución stock de $1000 \mathrm{mg} \mathrm{L}^{-1}$ de cada analito Merck. Se utilizó ácido nítrico $\left(\mathrm{HNO}_{3}\right)$ ppa. Merck para las curvas de calibración y para digestión de la muestra en diluciones de $0,028 \mathrm{~mol} \mathrm{~L}^{-1}(0,2 \% \mathrm{v} / \mathrm{v})$ y $10,5 \mathrm{~mol} \mathrm{~L}^{-1}(25 \% \mathrm{v} / \mathrm{v})$, con agua tipo 2 .

El modificador usado es fosfato de amonio monobásico $\left(\mathrm{NH}_{4} \mathrm{H}_{2} \mathrm{PO}_{4}\right)$, Carlos Erba. Para HG-AAS y CV-AAS, se utilizó borohidruro de sodio $\left(\mathrm{NaBH}_{4}\right)$ Aldrich, hidróxido de sodio $(\mathrm{NaOH})$ pa. Panreac y ácido clorhídrico $(\mathrm{HCl})$ pa. J. T. Baker. Para la reducción del As también se usó ioduro de sodio (NaI) Dolder.

\section{Muestra}

Para el desarrollo de la metodología se utilizó cabello donado por adultos sanos, no expuestos ocupacionalmente. Para evaluar algunas cifras de mérito se usó un material de referencia certificado, número 13 de cabello humano, del Instituto Nacional de Estudios Ambientales (NIES) de Japón.

\section{Parámetros analíticos y equipos}

La validación se realizó en el Centro Especializado de Química Toxicológica (CEQUIMTOX). La determinación de los elementos se hizo por espectrometría de absorción atómica. La determinación de Hg y As se llevó a cabo utilizando un equipo de espectrometría de absorción atómica Varian 55B, con un sistema de flujo de generación de vapor VGA 77 Agilent para cada elemento. La determinación de Hg se realizó por CV-AAS, y la de As por HG-AAS. Para la determinación se trabajó a 253,6 nm y a 193,7 nm, respectivamente.

$\mathrm{Mn}$ y $\mathrm{Pb}$ fueron analizados mediante ET-AAS utilizando un equipo de espectrometría de absorción atómica Thermo iCE serie 3000, con automuestreador y corrección de fondo Zeeman. La determinación se realizó en las líneas 283,3 nm y $279,5 \mathrm{~nm}$, respectivamente.

\section{Limpieza y digestión de las muestras}

La limpieza de las muestras fue adaptada de Menezes et al. (2009). Se tomó una muestra de pelo de la nuca, de aproximadamente $0,5 \mathrm{~cm}$ de diámetro, y se cortó lo más próximo posible al cuero cabelludo con una tijera quirúrgica de acero inoxidable. Se tomó el centímetro de pelo del extremo del cuero cabelludo y se lo colocó en baño de ultrasonido durante 20 minutos con TritonX-100 al 1\%m/v. La muestra se enjuagó con agua desionizada hasta que desapareciera la espuma. Luego se colocó nuevamente en un baño de ultrasonido durante 10 minutos con $\mathrm{HNO}_{3} 1 \mathrm{~N}$ y se enjuagó al menos tres veces con agua desionizada. A continuación se colocó 
en una estufa a $80{ }^{\circ} \mathrm{C}$ durante 24 horas y posteriormente se almacenó en una bolsa plástica hasta su digestión.

Para la digestión de las muestras se evaluó el contenido de $\mathrm{HNO}_{3}$ necesario para obtener una solución límpida y sin suspensiones. Se utilizaron diferentes concentraciones de $\mathrm{HNO}_{3}(100 \% \mathrm{v} / \mathrm{v}, 50 \% \mathrm{v} / \mathrm{v}$ y $25 \%$ v/v). Finalmente, la digestión se realizó con $25 \mathrm{mg}$ de pelo y $10 \mathrm{~mL}$ de $\mathrm{HNO}_{3} 25 \% \mathrm{v} / \mathrm{v}$, utilizando el método de digestión diseñado para cabellos incluido en el equipo de horno microonda Mars 6 , CEM. El programa de temperaturas se presenta en la Tabla 1.

TABLA 1. Temperaturas del programa Hair.

\begin{tabular}{|ccc|}
\hline Etapa & Tiempo (minutos) & Temperatura $\left({ }^{\circ} \mathbf{C}\right)$ \\
\hline Rampa de calentamiento & 15 & Hasta 180 \\
\hline Mantenimiento & 15 & 180 \\
\hline Enfriamiento & 15 & $40-50$ \\
\hline
\end{tabular}

\section{Parámetros de desempeño}

Debido a los niveles admitidos para los diferentes analitos se evaluó un rango lineal de 0 a $12,5 \mu \mathrm{g} \mathrm{L} \mathrm{L}^{-1}$ para cada elemento. Se realizaron fortificaciones antes de las digestiones para evaluar la veracidad, además del uso del material de referencia certificado. El modelo de mejor ajuste se evaluó mediante inspección visual, coeficiente de determinación $\left(\mathrm{R}^{2}\right)$ y la prueba lack of fitness (LOF). El efecto matriz fue evaluado con el material de referencia certificado para $\mathrm{Pb}, \mathrm{Hg}$ y Mn $(n=8)$. Dada la baja concentración del As en material de referencia, que no puede ser detectada por estas técnicas (EAA), la veracidad se evaluó mediante una prueba t-Student.

Los límites de cuantificación (LOQ) y límites de detección (LOD) se calcularon con los blancos $(n=10)$, utilizando el criterio 10s y 3s (Eurachem, 2014). La precisión como repetibilidad se evaluó en tres niveles de concentración $\left(3,6\right.$ y $9 \mu \mathrm{g} \mathrm{L} \mathrm{L}^{-1}$, $\mathrm{n}=10$ ). La precisión intermedia se estudió para la variable tiempo comparando los valores medios. La veracidad se evaluó con un material de referencia certificado para el caso de $\mathrm{Mn}$ y $\mathrm{Hg}(\mathrm{n}=8)$; para el $\mathrm{As} \mathrm{y} \mathrm{Pb}$ fue evaluada utilizando la recuperación en dos niveles diferentes de concentración $(n=2)$, debido a la baja cantidad de As en el material, y al efecto matriz en $\mathrm{Pb}$. La evaluación de la incertidumbre se realizó mediante el protocolo PLB05 del CEQUIMTOX, que establece que, en caso de contar con interlaboratorios, la incertidumbre (U) se calcula como el doble de la desviación estándar de la precisión intermedia. 


\section{RESULTADOS Y DISCUSIÓN}

\section{Condiciones CV-AAS}

Se probaron las condiciones de generación de vapor frío utilizadas habitualmente en el CEQUIMTOX para la determinación de $\mathrm{Hg}$ en orina. Como los resultados eran adecuados (estabilidad y altura máxima de la señal) se utilizaron las mismas condiciones: flujo: $6 \mathrm{~mL} \mathrm{~min}{ }^{-1}$, $\mathrm{HCl}: 40 \% \mathrm{v} / \mathrm{v}, \mathrm{NaBH}_{4} 0,6 \% \mathrm{~m} / \mathrm{v}$ en $\mathrm{NaOH} \mathrm{0,5 \%} \mathrm{m/v.}$ Debido a la toxicidad de $\mathrm{Hg}$ es necesario preparar una trampa de $\mathrm{Hg}$ con permanganato de potasio para la captura del vapor de $\mathrm{Hg}$.

\section{Condiciones HG-AAS}

$\mathrm{Al}$ igual que con CV-AAS, tanto la reducción de la muestra como las condiciones para la generación del hidruro se evaluaron por primera vez mediante la realización del protocolo utilizado habitualmente para la determinación de As en orina. Como los resultados fueron adecuados (estabilidad y altura máxima de la señal), las condiciones se mantuvieron: flujo: $6 \mathrm{~mL} \mathrm{~min}{ }^{-1}, \mathrm{HCl} 5 \mathrm{~N}, \mathrm{NaBH}_{4} 0,6 \% \mathrm{~m} / \mathrm{v}$ en $\mathrm{NaOH} 0,5 \% \mathrm{~m} / \mathrm{v}$. La reducción de As (V) a As (III) fue realizada agregando $1 \mathrm{~mL}$ de $\mathrm{NaI} 27 \% \mathrm{~m} / \mathrm{v}$ y $1 \mathrm{~mL}$ de $\mathrm{HCl}$ conc. cada $10 \mathrm{~mL}$ de muestra.

\section{Optimización de ET-AAS}

Las condiciones de ET-AAS se optimizaron para Mn y Pb. No se necesitó ningún modificador de matriz para $\mathrm{Mn}$, pero en el caso de $\mathrm{Pb}$ se utilizó fosfato de amonio de $5 \mathrm{~g} \mathrm{~L}^{-1}$. Las temperaturas óptimas de pirólisis y atomización fueron $800 / 1900{ }^{\circ} \mathrm{C}$, y $800 / 2200{ }^{\circ} \mathrm{C}$ para $\mathrm{Mn}$ y $\mathrm{Pb}$, respectivamente. En las Tablas 2 y 3 se presentan los programas de temperatura utilizados.

TABla 2. Programa de temperaturas mediante ET-AAS para la determinación de Mn.

\begin{tabular}{|ccccc|}
\hline Temperatura $\left({ }^{\circ} \mathbf{C}\right)$ & Tiempo (min) & Rampa & $\begin{array}{c}\text { Flujo de gas } \\
(\mathbf{L} / \mathbf{m i n})\end{array}$ & Etapa \\
\hline 100 & 30 & 10 & 0,2 & Secado \\
\hline 800 & 20 & 150 & 0,2 & Pirólisis \\
\hline 1900 & 3 & 0 & Apagado & Atomización \\
\hline 2000 & 3 & 0 & 0,2 & Limpieza \\
\hline
\end{tabular}


TABLA 3. Programa de temperaturas mediante ET-AAS para la determinación de $\mathrm{Pb}$.

\begin{tabular}{|ccccc|}
\hline Temperatura $\left({ }^{\circ} \mathbf{C}\right)$ & Tiempo (min) & Rampa & $\begin{array}{c}\text { Flujo de gas } \\
(\mathbf{L} / \mathbf{m i n})\end{array}$ & Etapa \\
\hline 95 & 10 & 4 & 0,2 & \\
\hline 100 & 20 & 4 & 0,2 & Secado \\
\hline 130 & 5 & 2 & 0,2 & \\
\hline 450 & 30 & 84 & 0,2 & Pirólisis \\
\hline 800 & 5 & 5 & 0,2 & Atomización \\
\hline 2200 & 2 & 0 & Apagado \\
\hline 2300 & 1 & 170 & 0,2 & Limpieza \\
\hline
\end{tabular}

\section{Rango de estudio}

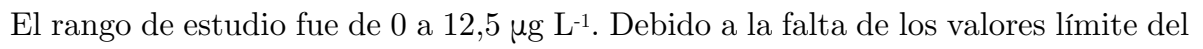
plomo en cabello se utilizaron como referencia los niveles encontrados en la región, de 6 a $8 \mu \mathrm{g} \mathrm{g-1}$. En el caso de As, $\mathrm{Hg}$ y Mn se utilizaron los valores de referencia antes mencionados, que están incluidos dentro del rango de estudio. La Figura 1 a-d muestra el mejor modelo de ajuste para cada elemento. $\mathrm{Pb}, \mathrm{Hg}$ y As tienen un comportamiento lineal, pero el Mn presenta un modelo del mejor ajuste cuadrático. La Tabla 4 presenta los resultados obtenidos luego de realizar el LOF; como los resultados obtenidos (U) son menores al F, los modelos de ajuste elegidos son correctos.

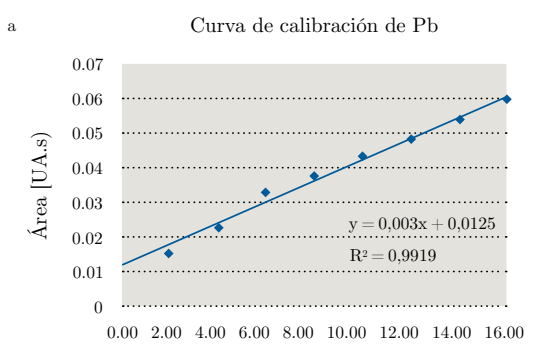

Concentración $\mathrm{Pb}$ en $\mu \mathrm{g} \mathrm{L}{ }^{-1}$

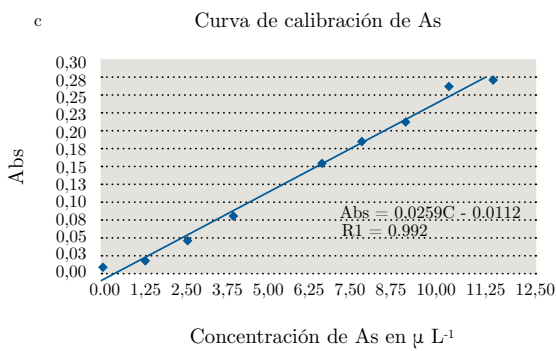

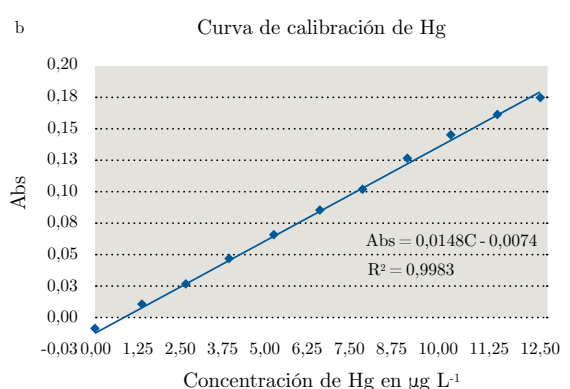

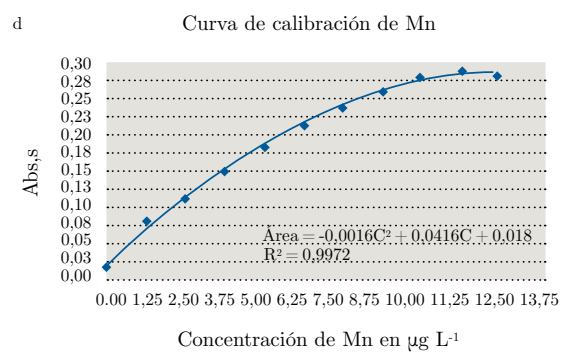

Figura 1. Curvas de calibración: a) Pb, b) Hg, c) As, d) Mn. 
TABLA 4. Prueba de mejor ajuste (LOF).

\begin{tabular}{ccccc} 
& As & Pb & Hg & Mn \\
\hline $\mathrm{U}$ & 2,444 & 2,571 & 2,333 & 2,750 \\
\hline $\mathrm{F}, 0,95$ & 3,049 & 3,160 & 3,049 & 3,049 \\
\hline
\end{tabular}

\section{Efecto matriz}

Con el fin de evaluar el efecto matriz del As, se realizó una prueba t-Student comparando la curva de calibración en $0,2 \% \mathrm{v} / \mathrm{v} \mathrm{HNO}_{3}$ y la curva realizada con cabello y adiciones estándar a lo largo del rango lineal de estudio $(n=3)$. Los resultados del test se presentan en la Tabla 5 e indican que no hay efecto matriz, por lo tanto, a efectos del análisis de rutina, la curva se realizará en esta disolución.

En el $\mathrm{Pb}$ se evaluó el efecto matriz utilizando el material de referencia certificado. El análisis se realizó en diferentes diluciones del material en $\mathrm{HNO}_{3} 0,2 \%$ por duplicado, en curva de calibración de la misma disolución. Los resultados presentados en la Tabla 6 muestran que los valores de recuperación obtenidos para las distintas diluciones no se encuentran dentro del rango de aceptación estipulado (75-125\% de recuperación), lo que evidencia el efecto matriz. Sin embargo, se realizó un estudio de coincidencia de matriz, matrix match $(\mathrm{n}=7)$, utilizando el test de Chow, que indica que no hay diferencia alguna entre los diferentes cabellos, por lo que para realizar la curva de calibración se deberá utilizar cabello de población no expuesta (Tabla 7).

TABLA 5. Prueba $t$ de dos colas para evaluar efecto matriz del As.

\begin{tabular}{cc}
$\boldsymbol{t}$-estadistico & $\boldsymbol{t}$-crítico \\
\hline 1,13646784 & 2,77644511 \\
\hline
\end{tabular}

Para $\mathrm{Hg}$ y Mn el efecto matriz también se evaluó utilizando el material de referencia certificado. Se hizo un duplicado de las diluciones y se interpoló en una curva de calibración en $\mathrm{HNO}_{3} 0,2 \%$, que se muestra en la Tabla 6 , donde se pueden ver los porcentajes de recuperación de las diferentes diluciones, mostrando que no se evidencia efecto matriz.

\section{Límites de detección (LOD) y de cuantificación (LOQ)}

LOD y LOQ se calcularon siguiendo los criterios 3s y 10s, según la guía Eurachem (Eurachem, 2014), donde s es la desviación estándar de 10 medidas de blanco. La Tabla 8 presenta los resultados obtenidos. Los límites de As, $\mathrm{Pb}$ y $\mathrm{Hg}$ son adecuados para el uso de esta metodología de screening. Sin embargo, para el caso del Mn, el límite de cuantificación obtenido está por encima del valor con el que se ha encontrado la aparición de efectos adversos de $3 \mu \mathrm{g} \mathrm{g}^{-1}$ de pelo en niños de Montreal (Menezes-Filho, et al., 2009). De todas formas, por ser la primera metodología 
desarrollada para la determinación de este metal en cabello del país, se utilizará como screening mientras se trabaja para disminuirlo.

TABLA 6. Evaluación del efecto matriz.

\begin{tabular}{cccccccc}
\hline & \multicolumn{2}{c}{ Mn } & \multicolumn{2}{c}{ Pb } & \multicolumn{2}{c}{ Hg } \\
\hline Nilución & \% REC & \% REC & \% REC & $\%$ REC & $\%$ REC & $\%$ REC \\
\hline $1 / 20$ & 87,80 & 96,15 & $-587,99$ & $-471,50$ & 50,72 & 93,84 \\
\hline $1 / 15$ & 131,71 & 115,38 & 17,26 & 43,63 & 86,81 & 99,40 \\
\hline $1 / 10$ & 97,56 & 105,77 & 45,27 & 57,22 & 95,04 & 108,83 \\
\hline $1 / 9$ & 96,59 & 108,17 & 24,54 & 50,32 & 112,01 & 118,84 \\
\hline $1 / 8$ & 109,27 & 115,38 & 44,60 & 63,03 & 113,19 & 121,11 \\
\hline $1 / 7$ & 105,85 & 111,06 & 43,82 & 66,82 & 116,38 & 122,23 \\
\hline $1 / 6$ & 105,37 & 98,08 & 38,91 & 60,82 & 122,05 & 121,79 \\
\hline $1 / 5$ & 100,00 & 105,77 & 23,85 & 50,08 & 129,58 & 116,97 \\
\hline $1 / 4$ & 101,46 & 100,00 & 47,59 & 66,47 & 126,58 & 118,85 \\
\hline $1 / 3$ & 93,66 & 100,96 & 49,28 & 68,99 & 131,63 & 106,94 \\
\hline $1 / 2$ & 81,95 & 78,85 & 45,70 & 59,47 & 132,66 & 95,03 \\
\hline
\end{tabular}

TABLA 7. Estadístico de Chow para evaluar efecto matriz del $\mathrm{Pb}$.

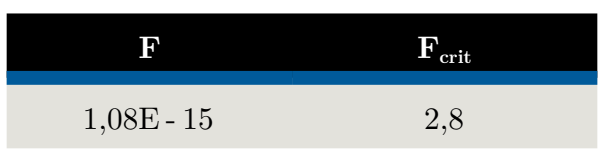

\section{Precisión}

La precisión como repetibilidad se determinó a tres niveles $\left(3,6,9 \mu \mathrm{g} \mathrm{L} \mathrm{L}^{-1}, \mathrm{n}=10\right)$ para As y $\mathrm{Hg}$. Para $\mathrm{Mn}$ y $\mathrm{Pb}$, se determinó a dos niveles $(6,9, \mathrm{n}=10$ para $\mathrm{Mn}$ y $\mathrm{n}=7$ para $\mathrm{Pb}$ ), debido a que el LOQ era superior a $3 \mu \mathrm{g} \mathrm{L} \mathrm{L}^{-1}$. Se evaluó utilizando el coeficiente de variación (CV\%) y los resultados que se muestran en Tabla 8 son adecuados para la metodología.

En el caso de la precisión intermedia, se realizó un test de comparación de valores medios.

\section{Veracidad}

Para $\mathrm{Mn}$ y $\mathrm{Hg}$, la veracidad se estimó utilizando el material de referencia NIES $(n=8)$, como se muestra en la Tabla 8 . La recuperación fue del 105,6\% y 108,1\%, 
respectivamente. En el caso del As, este parámetro se estimó usando muestras de cabello fortificadas $(n=4)$ a 3 y $6 \mu \mathrm{g} \mathrm{L} \mathrm{L}^{-1}$. La recuperación en este caso fue de 106,5\%. Debido al efecto de matriz en las curvas de calibración de $\mathrm{Pb}$, no fue posible determinar la veracidad utilizando el material de referencia. La recuperación se estimó utilizando muestras fortificadas $(\mathrm{n}=4)$ a diferentes niveles, 6,25 y $12,50 \mu \mathrm{g} \mathrm{L} \mathrm{L}^{-1}$. La recuperación obtenida para este analito fue del 91,9\%.

\section{Incertidumbre}

Se evaluó en la metodología para tres niveles de concentración distintos, los mismos que fueron utilizados para precisión: 3,6 y $9 \mu \mathrm{g} \mathrm{L}^{-1}$. Los resultados se encuentran en la Tabla 8.

TABLA 8. Parámetros de desempeño.

\begin{tabular}{|c|c|c|c|c|}
\hline Elemento & As & $\mathrm{Pb}$ & $\mathrm{Hg}$ & Mn \\
\hline LOD $(\mu g g-1)$ & 0,121 & 0,961 & 0,249 & 1,319 \\
\hline LOQ ( $\mu g$ g-1) & 0,403 & 3,204 & 0,830 & 3,860 \\
\hline Precisión $(\mathrm{CV} \%, \mathrm{n}=10)\left(3 \mu \mathrm{g} \mathrm{g}^{-1}\right)$ & 6,6 & - & 6,4 & - \\
\hline Precisión $(\mathrm{CV} \%, \mathrm{n}=10)\left(6 \mu \mathrm{g} \mathrm{g}^{-1}\right)$ & 7,0 & $10,9(\mathrm{n}=7)$ & 7,8 & 4,9 \\
\hline Precisión $(\mathrm{CV} \%, \mathrm{n}=10)\left(9 \mu \mathrm{g} \mathrm{g}^{-1}\right)$ & 5,5 & $6,4(\mathrm{n}=7)$ & 5,1 & 4,06 \\
\hline Veracidad (\% recuperación) & $106,5^{*}$ & $94,9^{* *}$ & $108,1^{* *}$ & $105,6^{*}$ \\
\hline \multirow{3}{*}{$\begin{array}{l}\text { Incertidumbre }\left(\mu \mathrm{g} \mathrm{L} \mathrm{L}^{-1}\right) \\
\quad\left(3,6,9 \mu \mathrm{g} \mathrm{L}^{-1}\right)\end{array}$} & 0,4 & - & 0,3 & - \\
\hline & 0,7 & 2,3 & 3,7 & 0,59 \\
\hline & 1,3 & 2,6 & 0,9 & 0,85 \\
\hline
\end{tabular}

* Material de referencia certificado Human Hair NIES; ** fortificaciones

\section{CONCLUSIONES}

Desde el punto de vista analítico, se logró desarrollar y validar un método de digestión y de determinación de cuatro elementos en una sola etapa de preparación de la muestra. Esto hace que se pueda contar con una herramienta analítica para estudios poblacionales en Uruguay.

Los elementos determinados en este estudio en la matriz cabello son ampliamente utilizados a nivel internacional como biomarcadores de exposición crónica, por lo cual se debe seguir avanzando e intentar mejorar los límites de detección y cuantificación, principalmente para el $\mathrm{Pb}$ y Mn en relación a los valores de referencia.

Se plantea como próximo desafío realizar un estudio preliminar en la población local para establecer los valores basales de estos elementos en cabello, propios de la población uruguaya. 


\section{REFERENCIAS}

Adams, L.B., Holloway, C.E., George, F. y Quig, D., 2006. Analyses of toxic metals and essential minerals in the hair of arizona children with autism and associated conditions, and their mothers. En: Biological Trace Element Research, 110(3), pp.193-209.

ATSDR, 2007. Toxicological profile for arsenic [En línea]. Atlanta: ATSDR.

[Consulta: 17 de noviembre, 2017]. Disponible en: https://www.atsdr.cdc.gov/ toxprofiles/tp2.pdf

ATSDR, 2017. CERCLA Priority list of hazardous substances [En línea]. Atlanta: ATSDR. [Consulta: 12 de marzo, 2019]. Disponible en: https://www.atsdr.cdc. gov/spl/previous/07list.html

ATSDR,2001. Summary report hair analysis panel discussion exploring the state of the scence [En línea]. Atlanta: ATSDR. [Consulta: 5 de noviembre, 2017]. Disponible en: https://www.ATSDR.cdc.gov/

Azevedo, F.A., de Souza Nascimento, E. y de MattaChasin, A. A., 2003. Mercúrio. En: Metais gerenciamento da toxicidade. [s.l.]: Atheneu.

Casarett, L.J. y Doull, J., 2005. Fundamentos de toxicología. Santiago de Compostela: Mc Graw-Hill/ Interamericana.

Colangelo, C. H., 2013. Mercurio. En: Rojas Martínez, Maritza, coord. Toxicología ambiental y ocupacional. Bogotá: Universidad del Rosario. pp. 237-256

Eurachem, 2014. The fitness for purpose of analytical methods. A laboratory guide to method validation and related topics [En línea]. 2a ed. Torino: Eurachem.

[Consulta: 15 de diciembre, 2017]. Disponible en: https://eurachem.org/index. $\mathrm{php} /$ publications/guides/mv

IARC, 2018. List of classification, volume 1-123 [En línea]. Lyon: IARC.

[Consulta: 3 de marzo, 2018] Disponible en: https://monographs.iarc.fr/list-ofclassifications-volumes/

Liang, G., Pan, L. y Liu, X., 2017. Assessment of typical heavy metals in human hair of different age groups and foodstuffs in Beijing, China. En: International Journal of Environmental Research and Public Health, 14(914), pp.1-10.

Mañay, N., 2013. Arsénico y cadmio. En: Rojas Martínez, Maritza coord. Toxicología ambiental y ocupacional. Bogotá: Universidad del Rosario. pp. 257-276

Marcano, E., Labady, M., Gomes, C., Aguiar, G. y Laine, J., 2009. Altos niveles de mercurio y plomo detectados por análisis de cabello en dos ambientes venezolanos. En: Acta Amazonica, 39(2), pp.315-318.

Menezes-Filho, J.A., Paes, C.R., de Pontes, A., Moreira J.C., Sarcinelli, P.N. y Mergler, D., 2009. High levels of hair manganese in children living in the vicinity of a ferromanganese alloy production plant. En: Neurotoxicology, 30(6), pp.1207-1213.

Naciones Unidas, 2010. Informe relativo a la información sobre los sistemas armonizados para medir la carga corporal de mercurio [En línea]. Estocolmo: UNEP. [Consulta: 25 de marzo, 2018]. Disponible en: http://www.mercuryconvention.org/Portals/11/documents/ meetings/inc2/spanish/INC2_6_s.doc 
Tirado Amador, L.R., González- Martínez, F.D., Martínes Hernández, L.J., Wilches Vergara, L. A. y Celedón-Suárez, J.N., 2015. Niveles de metales pesados en muestras biológicas y su importancia en salud. En: Rev. Nac. de Odont., 11(21), pp.1-30.

Tokar, E.J., Boyd, W.A., Freedman, J.H. y Waalkes, M.P., 2013. Toxic effects of metals. En: Toxicology. The basic science of poisons. 8va ed. Santiago de Compostela: Mc Graw-Hill.

Valdebenito, G. A., 2008. Desarrollo de un método para la determinación directa de $\mathrm{Pb}$ mediante espectrometría de absorción atómica electrotérmica (ETAAS) en suspensiones de pelo y uña («slurries") como biomarcadores de exposición [En línea]. [s.l.]: Universidad de Chile. (Tesis de pregrado). [Consulta: 11 de noviembre, 2017]. Disponible en: http://repositorio.uchile.cl/handle/2250/105699

WHO, 2015. Human biomonitoring: facts and figures. CopenhagEn: WHO Regional Office for Europe. 